Pacific Journal of Mathematics

ONE PARAMETER SUBSEMIGROUPS IN LOCALLY
COMPLETE DIFFERENTIABLE SEMIGROUPS 


\title{
ONE PARAMETER SUBSEMIGROUPS IN LOCALLY COMPLETE DIFFERENTIABLE SEMIGROUPS
}

\section{J. P. Holmes}

\begin{abstract}
We present here some theorems concerning the existence of one parameter subsemigroups in the generalized differentiable semigroups introduced by Graham. These semigroups are based on a type of generalized differentiable manifold and include as examples many semigroups of matrices and subsemigroups of Lie groups which are not ordinary manifolds. The multiplication function is required to be strongly differentiable (a generalization of Frechet differentiability). We shed some light on a question of Graham by showing that such locally complete $C^{k}$ monoids which contain $C^{2}$ curves starting at 1 must contain $C^{k}$ one parameter subsemigroups. The argument shows that closed submonoids of Lie groups which contain $C^{2}$ curves with one end at 1 contain one parameter subsemigroups.
\end{abstract}

Preliminaries. The notion of semigroup with differentiable multiplication based on an ordinary differentiable manifold was studied in [H1, H2]. If such a $C^{k}$ semigroup $S$ has an idempotent $e$ then there is an open subgroup of $e S e$ which has $e$ as its identity element [H2, Theorem 1]. Thus one may appeal to Lie theory for the existence of one parameter subsemigroups. Indeed, each one parameter subsemigroup is contained in a one parameter subgroup. This is not the case with the differentiable semigroups defined by Graham.

The definitions in this paragraph are due to Graham in [G1, G2]. A subset $A$ of the Banach space $E$ is said to be admissible provided that each point of $A$ is a limit point of the interior of $A$. A function, $f$, with domain the admissible set $A$ and range in the Banach space $F$ is differentiable at the point $p$ in $A$ provided that there is a continuous linear function $T$ from $E$ to $F$ so that for each positive number $c$ there is a positive number $d$ so that if each of $x$ and $y$ is within $d$ of $p$ then

$$
|f(x)-f(y)-T(x-y)|<c|x-y| \text {. }
$$

In this case $T$ is unique and is denoted by $f^{\prime}(p)$. As usual, the statement that $f$ is $C^{1}$ on $A$ means that $f$ is differentiable at each point of $A$ and the function $f^{\prime}$ is continuous as a function from $A$ into $L(E, F)$, the space of linear transformations from $E$ to $F$ with the usual norm topology. The statement that $f$ is $C^{k}$ means that $f^{(k-1)}$ is $C^{1}$. A Hausdorff topological space $S$ is a $C^{k}$ manifold based on the Banach 
space $E$ provided that for each point $p$ of $S$ there is a homeomorphism $g_{p}$ from a neighborhood $U$ of $p$ onto an admissible subset $A$ of $E$ containing 0 so that $g_{p}(p)=0$ and the composition $g_{p} \circ g_{q}^{-1}$ is $C^{k}$ on its domain for each choice of $p$ and $q$ in $S$. The theory of these manifolds including the definition of product manifold is elucidated in [G2]. Finally, according to Graham, a topological semigroup is said to be $C^{k}$ provided that it is based on a $C^{k}$ manifold and the multiplication function is $C^{k}$ as a function from $S \times S$ into $S$.

This notion of differentiable semigroup includes as examples such things as the closed interval $[0,1]$ under ordinary real multiplication, the unit disk $D$ under ordinary complex multiplication, and certain closed subsemigroups of Lie groups studied in $[\mathbf{H L}]$ which were excluded under the definition in [H1].

Much of the calculus on $C^{k}$ manifolds mimics the standard theory. An exception is the possible nonexistence of solutions to differential equations (vector fields may point outside of the manifold).

EXAMPLES. Example 1. A $C^{k}$ monoid need have no nontrivial one parameter subsemigroups. For example from [G1] let $S$ be the subset of the plane to which $(x, y)$ belongs if and only if $(x, y)$ is $(0,0)$ or $x>0$ and $y$ is between 0 and $x^{2} . S$ forms a $C^{k}$ monoid under vector addition for each positive $k$. There is no non-constant homomorphism from $R^{+}$ into $S$.

Example 2. Local $C^{k}$ groups must have some neighborhood of the identity element covered with one parameter subsemigroups (actually subroups). The following example shows that some differentiability is needed for this conclusion.

Let $S$ be the Banach space of continuous real valued functions on $R$ with limit at $+\infty$ and $-\infty$. For each $f$ and $g$ in $S$ define $f g$ by $f g(x)=f(g(x)+x)+g(x)$. This multiplication is obtained by mapping $f$ and $g$ to $f+$ id and $g+$ id, composing, then subtracting id from the result. Thus, $S$ is isomorphic to a subsemigroup of the semigroup under composition of continuous functions from $R$ to $R$. $S$ is a Banach space when equipped with the supremum norm. The multiplication is continuous but not differentiable relative to the Banach space topology. The constantly zero function is the only idempotent and is also an identity element.

There are elements arbitrarily close to the identity element which have no square root and hence are in no one parameter subsemigroup. To see this consider for a positive number $c$ the function $f$ defined by $f(x)=c$ if $x \leq 0, f(x)=-2 x$ if $x$ is in $[0, c / 2]$, and $f(x)=0$ if $x>c / 2$. It is fairly easy to see that if $g$ is in $S$ and $g g=f$ then $g(x)=c / 2$ if $x \leq 0$ and 
$g(x)=0$ if $x>c / 2$. But this implies that

$$
c=f(0)=g(g(0)+0)+g(0)=g(c / 2)+c / 2=c / 2 \text {. }
$$

thus there is no $g$ so that $g g=f$. These properties of this example are explicated in [H3].

There are also elements arbitrarily close to the identity element which are in uncountably many one parameter subgroups. To see this consider the isomorphism into the semigroup of functions from $R$ to $R$ noted above. If a function agrees with id outside the interval $[0, c](c>0)$ then it is in the image of the isomorphism.

Suppose $c>0$ and $f$ is a homeomorphism from $R$ to $R$ which agrees with id outside $(0, c)$ and so that $f(x)>x$ if $x$ is in $(0, c)$. We can build an increasing homeomorphism $g$ from $(0, c)$ onto $R$ satisfying $g(f(x))=$ $g(x)+1$ in the following way. Choose $a$ in $(0, c)$ and note that the sequence

$$
\ldots, f^{-2}(a), f^{-1}(a), a, f(a), f^{2}(a), \ldots
$$

is increasing and converges to 0 on the left and $c$ on the right. Define $g$ on $[a, f(a)]$ to be an arbitrary increasing homeomorphism onto $[0,1]$. There is exactly one way to define $g$ on $\left[f(a), f^{2}(a)\right]$ to make $g(f(x))=g(x)$ +1 for $x$ in $[a, f(a)]$ since each $y$ in $\left[f(a), f^{2}(a)\right]$ is $f(x)$ for exactly one $x$ in $[a, f(a)]$. This extension of $g$ is continuous, increasing, and maps $\left[a, f^{2}(a)\right]$ onto $[0,2]$. Continuing in this way we obtain a $g$ with the desired property. If $T$ is defined from $R$ into $S+$ id by $T(s)(x)=x$ if $x$ is not in $(0, c)$ and $T(s)(x)=g^{-1}(g(x)+s)$ if $x$ is in $(0, c)$ then the function $Q$ defined from $R$ into $S$ by $Q(s)=T(s)$ - id is a continuous isomorphism. The norm of each $Q(s)$ is no more than $c$ and

$$
Q(1)=f-\mathrm{id} \text {. }
$$

The arbitrariness of $g$ on $[a, f(a)]$ accounts for the existence of uncountably many such $Q$ 's. The author was introduced to this construction by Gordon Johnson.

Locally complete semigroups. We now turn our attention to $C^{k}$ monoids in which $k$ is at least 2 and which have a neighborhood, $U$, of 1 so that $g_{1}(U)$ is a closed subset of $E$. We say that such a monoid is locally complete. The main result is that if such a monoid contains a $C^{2}$ arc with one end at 1 then it has a $C^{k}$ one parameter subsemigroup. The examples show that some completeness near 1 is needed and indicate that some differentiability hypothesis may be needed. On the other hand we are not aware of a locally complete connected $C^{1}$ monoid with no one parameter subsemigroups. Our argument implies that if a closed submonoid of a Lie group contains a $C^{2}$ arc with one end at 1 then it contains a one parameter subsemigroup. 
TheOREM 1. Suppose $k \geq 2, S$ is a $C^{k}$ locally complete monoid, and there is a $C^{2}$ function, $h$, from $[0,1]$ into $S$ with $h(0)=1$. Then there is a $C^{k}$ function, $T$, from $R^{+}$to $S$ so that $T(s) T(t)=T(s+t)$ whenever each of $s$ and $t$ is in $R^{+}$and $T^{\prime}(0)=h^{\prime}(0)$.

In [N1] Neuberger showed for $C^{2}$ local groups that if $y$ is the solution of the initial value problem $y^{\prime}=L(y)$ and $y(0)=1$, for $L$ a left invariant vector field, then $y(t)$ can be approximated by products of scalar multiples of $L(1)$ with the coefficients adding to $t$. The existence of $y$ is assured because $L$ must be $C^{1}$. Of course this $y$ is a local one parameter subgroup of the local group.

In [M] Marsden gives sufficient conditions for the pointwise convergence of $K(t / n)^{n}$ where, for each $t, K(t)$ is a self map of the ordinary Banach manifold $M$ and composition is the operation used in forming $K(t / n)^{n}$. If we interpreted $K(t)$ as left multiplication by $h(t)$ (which is a one-to-one correspondence for $t$ near 0 ) we could obtain Theorem 1 as a consequence of a translation of Marsden's argument for [M, Theorem 2.1] to the present setting of generalized Banach manfolds. Our Lemmas 1.1 and 1.3 would serve as the non-trivial part of verifying that this version of $K$ satisfies a translated version of the hypotheses of [M, Theorem 2.1]. The argument we present here is more straightforward in the present setting and shows that left multiplication by $T(t)$ is approximated, for $t$ near 0 , by products of the form $K\left(t_{n}\right) K\left(t_{n-1}\right) \cdots K\left(t_{0}\right)$ where the $t_{i}$ 's are non-negative and sum to $t$.

In our setting, solutions to the initial value problem $y^{\prime}=L(y)$, $y(0)=1$ for $L$ a left invariant vector field are not guaranteed by standard existence and uniqueness theorems because such an $L$ might point outside of $S$. Thus, we resort to product integrals which stay in $S$ because $S$ is a semigroup.

The author appreciates the help of the referee in pointing out reference $[\mathbf{M}]$.

To formulate an argument choose a closed (in $E$ ) neighborhood, $D$, of 0 in $g_{1}(S)$ so that

$$
V(x, y)=g_{1}\left(g_{1}^{-1}(x) g_{1}^{-1}(y)\right)
$$

exists whenever each of $x$ and $y$ is in $D$. We may slow $h$ down if necessary so that it maps into $g_{1}^{-1}(D)$ and let $f$ be defined by $f(t)=$ $g_{1}(h(t))$ for each $t$ in $[0,1]$. Since $h$ is $C^{2}$ so is $f$. The first lemma is a sort of Taylor's theorem for $V$. 
LEMMA 1.1. There are positive numbers $M, r$, and $d$ so that if $s$ is in $[0, r], q$ is in $D$, and $|q| \leq d$ then

$$
V(f(s), q)=q+V^{\prime}(0, q)(f(s), 0)+A(s, q)
$$

where $|A(s, q)| \leq M s^{2}$ and

$$
|V(f(s), q)| \leq|q|+M\left(s+s^{2}\right) .
$$

Proof. Choose, by continuity of $V^{\prime}$ and $V^{\prime \prime}$ at $(0,0)$, positive numbers $d$ and $N$ so that if each of $p$ and $q$ is in $D$ and each has norm not exceeding $d$ then $\left|V^{\prime}(p, q)\right| \leq N$ and $\left|V^{\prime \prime}(p, q)\right| \leq N$. Choose $N^{\prime}$ so that $\left|f^{\prime}(t)\right| \leq N^{\prime}$ for each $t$ in $[0,1]$. Note that

$$
|f(x)-f(t)| \leq N^{\prime}|s-t|
$$

whenever each of $s$ and $t$ is in $[0,1]$. Choose $r$ in $(0,1)$ so that $r N^{\prime} \leq d$ and let $M=N N^{\prime 2}+N N^{\prime}$.

Suppose $s$ is in $[0, r], q$ is in $D$, an $|q| \leq d$. Then

$$
\begin{aligned}
V( & f(s), q)-q=V(f(s), q)-V(0, q) \\
= & \int_{0}^{1} d a\left[V^{\prime}(f(0), q)\left(f^{\prime}(a s)(s), 0\right)\right] \\
& +\int_{0}^{1} d a\left[V^{\prime}(f(a s), q)-V^{\prime}(f(0), q)\right]\left(f^{\prime}(a s)(s), 0\right) \\
= & V^{\prime}(0, q)(f(s)-f(0), 0) \\
& +\int_{0}^{1} d a \int_{0}^{1} d b\left[V^{\prime \prime}(f(\text { bas }), q)\left(\left(f^{\prime}(a s)(s), 0\right),\left(f^{\prime}(\text { bas })(a s), 0\right)\right)\right] \\
= & V^{\prime}(0, q)(f(s), 0)+A(s, q) .
\end{aligned}
$$

By choice of $r, \mid f($ bas $) \mid \leq N^{\prime}$ bas $\leq d$ if each of $a$ and $b$ is in $[0,1]$ and moreover each of $\left|f^{\prime}(a s)(s)\right|$ and $\mid f^{\prime}($ bas $)(s) \mid$ is bounded by $N^{\prime} s$. Hence the integrand in $A(s, q)$ is bounded in norm by $N^{\prime 2} s^{2}$. It follows that $|A(s, q)| \leq M s^{2}$. Finally, since $|q| \leq d$ and $|f(s)| \leq s N^{\prime}$, we have

$$
|V(f(s), q)| \leq|q|+M\left(s+s^{2}\right) .
$$

COROLlaRY 1.2. There are positive numbers $B$ and e so that if each of $s_{1}, \ldots, s_{n}$ is a positive number and $a=\sum_{1}^{n} s_{i} \leq e$ then

$$
V\left(f\left(s_{n}\right), V\left(f\left(s_{n-1}\right), \ldots, V\left(f\left(s_{2}\right), f\left(s_{1}\right)\right) \ldots\right)\right)
$$

exists and has norm not exceeding $a B$.

Proof. Choose $M, r$, and $d$ as in Lemma 1.1, let $B=2 M$, and choose $e$ positive so that $e \leq r$ and $e B \leq d$. Suppose each of $s_{1}$ and $s_{2}$ is positive and $s_{1}+s_{2} \leq e$. Each of $s_{2} \leq r$ and $f\left(s_{1}\right)$ has norm not exceeding $d$ so by Lemma 1.1 we have

$$
\left|V\left(f\left(s_{2}\right), f\left(s_{1}\right)\right)\right| \leq s_{2}\left(M+s_{2} M\right)+\left|f\left(s_{1}\right)\right| \leq B\left(s_{2}+s_{1}\right) .
$$


Assume that $n$ is an integer which is at least 2 and suppose inductively that if and each of $s_{1}, \ldots, s_{n}$ is positive and $b=\sum_{1}^{n} s_{i} \leq e$ then $q=V\left(f\left(s_{n}\right), V\left(f\left(s_{n-1}, \ldots, V\left(f\left(s_{2}\right), f\left(s_{1}\right)\right) \ldots\right)\right)\right.$ exists and has norm not exceeding $b B$.

Suppose that each of $s_{1}, \ldots, s_{n+1}$ is positive and $a=\sum_{1}^{n+1} s_{i} \leq e$. By the inductive hypothesis we have the existence of $q$ as above with norm not exceeding $B b \leq B e \leq d$. Moreover, $s_{n+1}$ is in $[0, r]$ so Lemma 1.1 implies that

$$
\left|V\left(f\left(s_{n+1}\right), q\right)\right| \leq s_{n+1}\left(M+s_{n+1} M\right)+|q| \leq a B .
$$

LEMMA 1.3. There are positive numbers $a, b$, and $C$ so that if $p$ is in $D$ and $|p| \leq b$ and each of $u, s$, and $t=u+s$ is in $[0, a]$ then

$$
|V(f(t), p)-V(f(s), V(f(u), p))| \leq C s t .
$$

Proof. Choose $M, r$, and $d$ as in Lemma 1.1. Choose $N^{\prime \prime}$ to be an upper bound for $\left|f^{\prime \prime}(x)\right|$ for each $x$ in $[0,1]$. Choose $a^{\prime}$ positive so that if each of $x, y, p$, and $q$ is in $D$ and each has norm not exceeding $a^{\prime}$ then

$$
\left|V^{\prime}(x, y)-V^{\prime}(p, q)-V^{\prime \prime}(0,0)(x-p, y-q)\right| \leq|(x-p, y-q)| \text {. }
$$

Choose $b$ positive and less than each of $a^{\prime} / 2$ and $d / 2$. Choose the number $a$ positive and less than $r$ so that $2 a M$ is less than each of $b$ and $r$ and so that $a N^{\prime}$ is less than $a^{\prime}$. Choose $C$ so that $C / 3$ exceeds each of $\left[\left|V^{\prime \prime}(0,0)\right|+1\right] 2\left[N^{\prime}+M\right] N^{\prime}, M N^{\prime \prime}$, and $M$.

If $p$ is in $D,|p| \leq b$, and each of $u, s$, an $t=u+s$ is in [0,a] then from Lemma 1.1 we have $|V(f(u), p)| \leq|p|+2 M u$ which by choice of $a$ and $b$ is smaller than each of $d$ and $a^{\prime}$.

Suppose $p$ is in $D,|p| \leq b$, and each of $u, s$, and $t=u+s$ is in $[0, a]$. Then

$$
\begin{aligned}
& |V(f(t), p)-V(f(s), V(f(u), p))| \\
& \quad=|V(f(t), p)-V(f(u), p)+V(f(u), p)-V(f(s), V(f(u), p))| .
\end{aligned}
$$

Since $u$ is in $[0, r]$ and $|V(f(u), p)| \leq d$ we have by Lemma 1.1 that the last term is

$$
\begin{aligned}
= & \mid \int_{0}^{1} d v\left[V^{\prime}\left(f^{\prime}(u+v s), p\right)\left(f^{\prime}(u+v s)(s), 0\right)\right] \\
& -\int_{0}^{1} d v\left[V^{\prime}(0, V(f(u), p))\left(f^{\prime}(v s)(s), 0\right)\right]-A(s, V(f(u), p)) \mid \\
\leq & \left|\int_{0}^{1} d v\left[V^{\prime}(f(u+v s), p)-V^{\prime}(0, V(f(u), p))\right]\left(f^{\prime}(u+v s)(s), 0\right)\right| \\
& +\left|\int_{0}^{1} d v\left[V^{\prime}\left(0, V(f(u), p)\left(f^{\prime}(u+v s)-f^{\prime}(v s)\right)(s), 0\right)\right]\right| \\
& +|A(s, V(f(u), p))| .
\end{aligned}
$$


We consider the three summands separately. For each $v$ in $[0,1]$ each of $V(f(u), p), p$, and $f(u+v s)$ has norm not excceding $a^{\prime}$ so

$$
\begin{aligned}
& \left|\left[V^{\prime}(f(u+v s), p)-V^{\prime}(0, V(f(u), p))\right]\left(f^{\prime}(u+v s)(s), 0\right)\right| \\
& \quad \leq\left[\left|V^{\prime \prime}(0,0)\right|+1\right][|f(u+v s)|+|p-V(f(u), p)|] N^{\prime} s \\
& \quad \leq\left[\mid V^{\prime \prime}(0,0)+1\right]\left[N^{\prime} t+N^{\prime} u+2 M u\right] N^{\prime} s \\
& \quad \leq\left[\mid V^{\prime \prime}(0,0)+1\right] 2\left[N^{\prime}+M\right] N^{\prime} s t \leq(C / 3) s t .
\end{aligned}
$$

Thus the first summand does not exceed $(C / 3)$ st. The second summand is, since $|V(f(u), p)| \leq d$,

$$
\begin{aligned}
& \mid V^{\prime}\left(0, V(f(u), p)\left(\int_{0}^{1} d v \int_{0}^{1} d w\left[f^{\prime \prime}(w u+v s)(u, s)\right], 0\right) \mid\right. \\
& \quad \leq M N^{\prime \prime} u s \leq(C / 3) s t .
\end{aligned}
$$

Finally, since $s$ is in $[0, r]$ and $|V(f(u), p)| \leq d$,

$$
|A(s, V(f(u), p))| \leq M s s \leq(C / 3) s t .
$$

By a subdivision, $H$, of the interval $[0, x]$ we mean a finite non-decreasing sequence $0=t_{1} \leq t_{2} \leq \cdots \leq t_{n}=x$. By $\operatorname{mesh}(H)$ we mean the length of the largest interval formed by adjacent $t$ 's. Finally, by $P(H)$ we mean the product

$$
V\left(f\left(t_{n}-t_{n-1}\right), \ldots, V\left(f\left(t_{3}-t_{2}\right), f\left(t_{2}-t_{1}\right)\right) \ldots\right)
$$

provided it exists. The next lemma provides for convergence of the $P(H)$ 's.

COROLlaRY 1.4. There are positive numbers $F$ and $c$ so that if $x$ is in $[0, c], H$ is a subdivision of $[0, x]$, and $H^{\prime}$ refines $H$ then each of $P(H)$ and $P\left(H^{\prime}\right)$ exist and $\left|P(H)-P\left(H^{\prime}\right)\right| \leq F \operatorname{mesh}(H)$.

Proof. Let $B$ and $e$ be chosen as in Corollary 1.2 and $a, b$, and $C$ be chosen as in Lemma 1.3. Choose $a^{\prime}>0$ as in the proof of Lemma 1.3 so that if each of $x, y, p$, and $q$ is in $D$ and has norm not exceeding $a^{\prime}$ then

$$
\begin{aligned}
& \left|V(x, y)-V(p, q)-V^{\prime}(0,0)(x-p, y-q)\right| \\
& \quad \leq|(x-p, y-q)| .
\end{aligned}
$$

Note that for such $x, y, p$, an $q$ we have

$$
|V(x, y)-V(p, q)| \leq G|(x-p, y-q)|
$$

where $G=\left|V^{\prime}(0,0)\right|+1$. Choose $c$ not exceeding each of $e$ and $a$ so that $B c$ is smaller than each of $b$ and $a^{\prime}$ and let $F=G C c$. 
Suppose $x$ is in $[0, c]$,

$$
H=\left\{t_{1}, \ldots, t_{n}\right\}
$$

is a subdivision of $[0, x]$, and $s$ is between $t_{i}$ and $t_{i-1}$ for some $i$ between 0 and $n$. The product, $P(H)$, exists by Corollary 1.2. Let $u_{j}=t_{j}-t_{j-1}$ for $j=1, \ldots, n, v=s-t_{i-1}$, and $w=t_{i}-s$. Note that by choice of $c$ each of $v, w$, and $u_{i}=v+w$ is in $[0, a]$. Let

$$
p=V\left(f\left(t_{i-1}-t_{i-2}\right), \ldots, V\left(f\left(t_{3}-t_{2}\right), f\left(t_{2}-t_{1}\right)\right) \ldots\right)
$$

and

$$
q=V\left(\left(t_{n}-t_{n-1}\right), \ldots, V\left(f\left(t_{i+2}-t_{i+1}\right), f\left(t_{i+1}-t_{i}\right)\right) \ldots\right) .
$$

By Corollary $1.2|p| \leq B t_{i-1} \leq b, \quad|V(f(u), p)| \leq B s \leq a^{\prime}$, $|V(f(w), V(f(v), p))| \leq B t_{i} \leq a^{\prime}$, and $|q| \leq B\left(t_{n}-t_{i}\right) \leq a^{\prime}$.

Note that $P(H)=V\left(q, V\left(f\left(u_{i}\right), p\right)\right)$. We wish to compare this with $Q=V(q, V(f(w), V(f(v), p)))$.

$$
\begin{aligned}
& |P(H)-Q| \quad \text { by choice of } a^{\prime} \\
& \quad \leq G\left|V\left(f\left(u_{i}\right), p\right)-V(f(w),(f(v), p))\right| \quad \text { by Lemma } 1.3 \\
& \quad \leq M C u_{i} w=(F / c) u_{i} w .
\end{aligned}
$$

Now suppose $H^{\prime}$ refines $H$. Let $s_{1}, \ldots, s_{j}$ be an increasing sequence of numbers so that $H^{\prime}=H \cup\left\{s_{1}, \ldots, s_{j}\right\}$. Let $H^{\prime}=H_{j}, H=H_{0}$ and

$$
H_{i}=H_{i+1}-\left\{s_{i+1}\right\}
$$

for $i=j, \ldots, 1$ and let $r_{i}$ be the next larger member of $H_{i}$ to $s_{i}$ for $i=1, \ldots, j$. The intervals $\left[s_{i}, r_{i}\right]$ are nonoverlapping and fill up less than $[0, c]$. The mesh of each $H_{i}$ is no larger than $\operatorname{mesh}(H)$. Thus we have

$$
\begin{aligned}
& \left|P\left(H^{\prime}\right)-P(H)\right| \leq \sum_{1}^{j}\left|P\left(H_{i}\right)-P\left(H_{i-1}\right)\right| \\
& \quad \leq(F / c) \operatorname{mesh}(H) \sum_{1}^{j}\left(r_{i}-s_{i}\right) \leq F \operatorname{mesh}(H) .
\end{aligned}
$$

Now it is clear by completeness of $D$ that for each $t$ in $[0, c]$ there is a unique $R(t)$ in $D$ so that if $\varepsilon$ is a positive number there is a positive number $\delta$ so that if $H$ is a subdivision of $[0, t]$ with mesh less than $\delta$ then $P(H)$ is within $\varepsilon$ of $R(t)$. From Corollary 1.2 we have $|R(t)| \leq B t$ and from construction it is clear that $V(R(t), R(s))=R(t+s)$ whenever each of $s, t$, and $s+t$ is in $[0, c]$. From these observations and continuity of $V$ we have $R$ continuous on $[0, c]$. 
We comment that associativity was used in the proof of Corollary 1.4 for convenience and the proof of the existence of the product integral $R(t)$ can be carried out for nonassociative $V$. Of course, in the nonassociative situation we do not obtain a local homomorphism of $[0, c]$ into $D$.

Let $T$ be defined on $[0, c]$ into $S$ by $T(t)=g_{1}^{-1}(R(t))$. It is easy to see that there is a unique extension of $T$ to $R^{+}$which is continuous. It remains to show that $T$ is $C^{k}$ and $T^{\prime}(0)$ can be made to be $h^{\prime}(0)$.

Lemma 1.5. With $f$ and $R$ defined above, $R$ is $C^{k}$ and $R^{\prime}(0)=f^{\prime}(0)$.

Proof. Suppose $c^{\prime}$ is a positive number and choose $d$ positive so that if each of $p$ and $q$ is in $D \times D$ and each of $p$ and $q$ is within $d$ of $(0,0)$ then

$$
\left|V(p)-V(q)-V^{\prime}(0,0)(p-q)\right|<c^{\prime}|p-q| .
$$

If each of $x$ and $y$ is in $D$ and each is within $d$ of 0 then

$$
\left|x-y-V^{\prime}(0,0)(x-y, 0)\right|<c^{\prime}|x-y| \text {. }
$$

Since 0 is a limit point of the interior of $D, x-y$ can be taken in any direction. This implies that $V^{\prime}(0,0)(x, 0)=V^{\prime}(0,0)(0, x)=x$ for each $x$ in $E$.

Suppose $a$ is a positive number, choose $M$ an upper bound for $\left|f^{\prime}(x)\right|$ for $x$ in $[0,1]$, choose $F$ and $c$ as in Corollary 1.4, and choose $B$ and $e$ as in Corollary 1.2.

Using differentiability of $V$ at $(0,0)$ and using $V^{\prime}(0,0)(x, y)=x+y$ choose $d$ positive so that if each of $p$ and $q$ is in $D$ and each has norm not exceeding $d$ then

$$
|V(p, q)-V(0, q)-p| \leq(a / 3 M)|p| .
$$

Choose $t$ positive and less than each of $c, e$, and $d / B$ and choose $N$ so that if $n \geq N$ then $3 F / n<a$. Choose such an $n$ and for each $i=1, \ldots, n$ let

$$
q_{i}=V(f(t / n), \ldots, V(f(t / n), f(t / n)) \ldots)
$$

where the product has $i$ factors.

Note that $q_{n}=P(H)$ where $H$ is the subdivision of $[0, t]$ with $n$ equal length subintervals. It follows from the choice of $t$ in $[0, e]$ that each $q$ has norm less than $B t$ which is smaller than $d$. It follows from the choice of $F$ and $c$ that since $t$ is in $[0, c]$ we have

$$
\left|R(t)-q_{n}\right| \leq F t / n<a t / 3 \text {. }
$$


But

$$
\begin{aligned}
\left|q_{n}-n f(t / n)\right| \leq & \left|V\left(f(t / n), q_{n-1}\right)-q_{n-1}-f(t / n)\right| \\
& +\left|V\left(f(t / n), q_{n-2}\right)-q_{n-2}-f(t / n)\right| \\
& \cdots \\
& +|V(f(t / n), f(t / n))-f(t / n)-f(t / n)| \\
\leq & n(a / 3 M)|f(t / n)| \leq a t / 3 .
\end{aligned}
$$

Thus we have for each $n \geq N$ and each $t$ sufficiently small that

$$
|R(t)-n f(t / n)|<a t .
$$

Hence $R^{\prime}(0)$ exists and is $f^{\prime}(0)$. Since $R(t+s)=V(R(t), R(s))$ for $t$ in $[0,1)$ and $s$ sufficiently small the chain rule implies that $R^{\prime}(t)$ exists for $t$ in $[0,1)$ and

$$
R^{\prime}(t)=V^{\prime}(R(t), 0)\left(0, R^{\prime}(0)\right)
$$

Thus $R$ is $C^{k}$ on $[0,1)$. This implies that $T$ is $C^{k}$ on $R^{+}$and we are done with Theorem 1 .

COROLlaRY 1.6. If $S$ is a closed submonoid of a $C^{k}$ group with $k$ at least 2 and $S$ contains a $C^{2}$ curve with one end at 1 then $S$ has a $C^{k}$ one parameter subsemigroup.

This is immediate from the above argument. The product integral may be carried out in the group but the fact that $S$ is a closed set which is closed under products implies that the one parameter subsemigroup constructed maps into $S$.

In [HL] Hofmann and Lawson study certain subsemigroups of Lie groups which they term Lie semigroups. They are $C^{k}$ monoids in the present sense. These monoids are generated by one parameter subsemigroups and the structure of such a monoid is connected with that of its Lie wedge (an analogue of Lie algebra in this setting). In view of Theorem 1 one might hope to obtain geometric conditions on a $C^{k}$ monoid which imply that it is a Lie semigroup in this sense.

\section{REFERENCES}

[G1] G. Graham, Differentiable Semigroups, Lecture Notes in Mathematics \#998, Springer-Verlag (1983), 57-127.

[G2] , Differentiable manifolds with generalized boundary, Czechoslovak Math. J., 34 (109) (1984), 46-63. 
[H1] J. P. Holmes, Differentiable semigroups, Colloquium Mathematicum vXXXII (1974), 99-104.

[H2] , Rees products in differentiable semigroups, Semigroup Forum, 25 (1982), 145-152.

[H3] _ Partial groupoids on a Banach space, $\mathrm{Ph}$. D. dissertation, Emory University, Atlanta, Georgia 1970.

[H4] _ Continuous homomorphisms are differentiable, Proc. Amer. Math. Soc., 65 (1977), 277-281.

[HL] K. H. Hofmann and J. D. Lawson, Foundations of Lie Semigroups, Lecture Notes in Mathematics \# 998, Springer-Verlag (1983), 128-201.

[M] J. Marsden, On product formulas for nonlinear semigroups, J. Functional Anal., 13 (1973), 51-72.

[N1] J. W. Neuberger, Differentiable Semigroups with a neighborhood of the identity homeomorhic to a Banach space, Proc. Amer. Math. Soc., 34 (1972), 595-600.

Received January 27, 1986.

AUBURN UNIVERSITY

AUBURN, AL 36849-3501 



\title{
PACIFIC JOURNAL OF MATHEMATICS EDITORS
}

\author{
V. S. VARADARAJAN \\ (Managing Editor) \\ University of California \\ Los Angeles, CA 90024 \\ HERBERT Clemens \\ University of Utah \\ Salt Lake City, UT 84112 \\ R. FINN \\ Stanford University \\ Stanford, CA 94305
}

\author{
HERMANN FLASCHKA \\ University of Arizona \\ Tucson, AZ 85721 \\ RAMESH A. GANGOLLI \\ University of Washington \\ Seattle, WA 98195 \\ VAUGHAN F. R. JONES \\ University of California \\ Berkeley, CA 94720 \\ ROBION KIRBY \\ University of California \\ Berkeley, CA 94720
}

\author{
C. C. Moore \\ University of California \\ Berkeley, CA 94720
}

H. SAMELSON

Stanford University

Stanford, CA 94305

HAROLD STARK

University of California, San Diego

La Jolla, CA 92093

\section{ASSOCIATE EDITORS}

\author{
R. ARENS \\ E. F. BECKENBACH \\ B. H. NEUMANN \\ F. WOLF \\ K. YOSHIDA \\ (1906-1982)

\section{SUPPORTING INSTITUTIONS} \\ UNIVERSITY OF ARIZONA \\ UNIVERSITY OF BRITISH COLUMBIA \\ CALIFORNIA INSTITUTE OF TECHNOLOGY \\ UNIVERSITY OF CALIFORNIA \\ MONTANA STATE UNIVERSITY \\ UNIVERSITY OF NEVADA, RENO \\ NEW MEXICO STATE UNIVERSITY \\ OREGON STATE UNIVERSITY

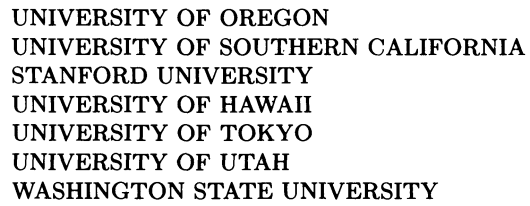

The Supporting Institutions listed above contribute to the cost of publication of this Journal, but they are not owners or publishers and have no responsibility for its content or policies.

Mathematical papers intended for publication in the Pacific Journal of Mathematics should be in typed form or offset-reproduced (not dittoed), double spaced with large margins. Please do not use built up fractions in the text of the manuscript. However, you may use them in the displayed equations. Underline Greek letters in red, German in green, and script in blue. The first paragraph must be capable of being used separately as a synopsis of the entire paper. In particular it should contain no bibliographic references. Please propose a heading for the odd numbered pages of less than 35 characters. Manuscripts, in triplicate, may be sent to any one of the editors. Please classify according to the scheme of Math. Reviews, Index to Vol. 39. Supply name and address of author to whom proofs should be sent. All other communications should be addressed to the managing editor, or Elaine Barth, University of California, Los Angeles, California 90024.

There are page-charges associated with articles appearing in the Pacific Journal of Mathematics. These charges are expected to be paid by the author's University, Government Agency or Company. If the author or authors do not have access to such Institutional support these charges are waived. Single authors will receive 50 free reprints; joint authors will receive a total of $\mathbf{1 0 0}$ free reprints. Additional copies may be obtained at cost in multiples of 50 .

The Pacific Journal of Mathematics is issued monthly as of January 1966. Regular subscription rate: $\$ 190.00$ a year (5 Vols., 10 issues). Special rate: $\$ 95.00$ a year to individual members of supporting institutions.

Subscriptions, orders for numbers issued in the last three calendar years, and changes of address should be sent to Pacific Journal of Mathematics, P.O. Box 969, Carmel Valley, CA 93924, U.S.A. Old back numbers obtainable from Kraus Periodicals Co., Route 100, Millwood, NY 10546.

The Pacific Journal of Mathematics at P.O. Box 969, Carmel Valley, CA 93924 (ISSN 0030-8730) publishes 5 volumes per year. Application to mail at Second-class postage rates is pending at Carmel Valley, California, and additional mailing offices. Postmaster: send address changes to Pacific Journal of Mathematics, P.O. Box 969, Carmel Valley, CA 93924.

PUBLISHED BY PACIFIC JOURNAL OF MATHEMATICS, A NON-PROFIT CORPORATION Copyright (C) 1987 by Pacific Journal of Mathematics 


\section{Pacific Journal of Mathematics}

Vol. 128 , No. $2 \quad$ April, 1987

Pierre Barrucand, John Harold Loxton and Hugh C. Williams, Some

explicit upper bounds on the class number and regulator of a cubic field

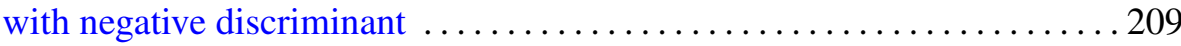

Thomas Ashland Chapman, Piecewise linear fibrations $\ldots \ldots \ldots \ldots \ldots 223$

Yves Félix and Jean-Claude Thomas, Extended Adams-Hilton's

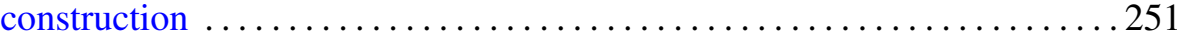

Robert Fitzgerald, Derivation algebras of finitely generated Witt rings . . . 265

K. Gopalsamy, Oscillatory properties of systems of first order linear delay differential inequalities ................................ 299

John P. Holmes, One parameter subsemigroups in locally complete

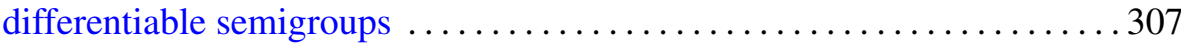

Douglas Murray Pickrell, Decomposition of regular representations for

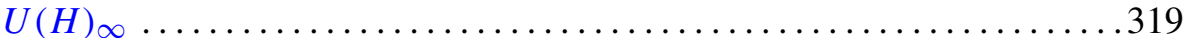

Victoria Powers, Characterizing reduced Witt rings of higher level

Parameswaran Sankaran and Peter Zvengrowski, Stable parallelizability

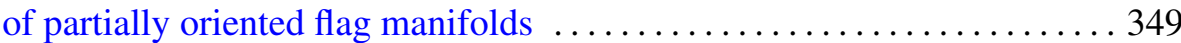

Johan Tysk, Eigenvalue estimates with applications to minimal surfaces . . . 361 Akihito Uchiyama, On McConnell's inequality for functionals of subharmonic functions

Minato Yasuo, Bott maps and the complex projective plane: a construction

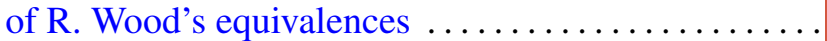

James Juei-Chin Yeh, Uniqueness of strong solutions to stochastic differential equations in the plane with deterministic boundary process 